\title{
The potential of corticomuscular and intermuscular coherence for research on human motor control
}

\author{
Tjeerd W. Boonstra ${ }^{1,2,3 *}$ \\ School of Psychiatry, University of New South Wales, Sydney, NSW, Australia \\ ${ }^{2}$ Black Dog Institute, Sydney, NSW, Australia \\ ${ }^{3}$ MOVE Research Institute, VU University, Amsterdam, Netherlands \\ *Correspondence: t.boonstra@unsw.edu.au \\ Edited by: \\ Sven Bestmann, University College London, UK \\ Reviewed by: \\ Simon Farmer, National Hospital for Neurology and Neurosurgery and Institute of Neurology, UK \\ Keywords: coherence analysis, oscillatory activity, motor neurons, EMG, descending pathways, computational modeling
}

In an experimental study on the changes in descending drive during muscle fatigue, Semmler et al. (2013) investigated intermuscular (EMG-EMG) coherence in elbow muscles after eccentric exercise. They reported a broadband increase in coherence with fatigue in all elbow flexor muscle pairs and suggested that these changes reflect increased common oscillatory input to the elbow flexors. This interpretation was questioned in the Editorial by Heroux and Gandevia (2013), who noted that the mechanisms generating the reported increase in coherence are unclear and that peripheral factors such as EMG signal cancellation may provide an alternative explanation for the observed changes in coherence. Héroux and Gandevia therefore, concluded that the insights that can be obtained from investigating corticomuscular (CMC) and intermuscular (IMC) coherence are limited.

In this commentary, I will first revisited the reasons for investigating IMC and CMC. These measures have the potential to provide key insights into the composition of descending drive (Farmer, 1998) and involve non-invasive recording techniques that make them suitable for clinical applications. Subsequently, I will review current research in this area aimed to uncover the generating mechanisms of IMC and CMC. In particular, the use of computational modeling and new recording techniques are considered to obtain a more accurate estimate of the underlying changes in descending drive.

CMC and IMC can provide important insights into the diverging projects underlying motor coordination. A major open questions in motor control is how the central nervous system (CNS) controls the redundant degrees of freedom of the musculoskeletal system (Bernstein, 1967). The possibility that the CNS produces movement through the flexible combination of groups of muscles, or muscle synergies, has received much attention (Todorov, 2004; Tresch and Jarc, 2009). However, the neural implementation of such control strategies remains largely unknown. IMC is particularly suited to investigate common input to groups of muscles and can be assessed in relatively unstrained and natural task settings. Several studies have explored this notion and showed that disparate muscles, whose activity needs to be coordinated, indeed reveal significant IMC (Boonstra et al., 2009a; Poston et al., 2010; Nazarpour et al., 2012). CMC and IMC can also be used to differentiate between pathways converging onto the spinal motoneurons. Common oscillatory input underlying IMC can originate from many efferent and afferent pathways, while CMC is most likely driven exclusively by the corticospinal pathway. By comparing IMC and CMC, one may distinguish cortical from non-cortical sources of common input (Boonstra et al., 2009b).

The mechanisms underlying CMC and IMC are still being discussed and their detailed understanding would greatly enhance their research potential. Computational modeling of the involved circuitry can be used to quantify the effects and involvements of potential mechanisms. The debate about EMG rectification is a good example of the strength of such a combined approach: Using converging empirical and computation evidence the specific contribution of peripheral mechanisms such as EMG signal cancellation can be determined (Boonstra and Breakspear, 2012; Farina et al., 2013; Ward et al., 2013). Likewise, combining experimental investigations with computational modeling can be used to identify the involved neural circuitry, e.g., to specify the spinal circuitry involved in the cancellation of $10-\mathrm{Hz}$ oscillations in corticospinal drive (Williams et al., 2010). Although invasive recordings are essential to validate the model design, once such a model is formulated model inversion techniques can be used to infer "hidden" model parameters (Friston et al., 2003), e.g., to infer common input from empirically observed CMC and IMC.

Computational models of CMC and IMC provide a quantitative description of the synaptic transformations of the involved pathways. CMC poses additional challenges concerning the transformation of synchronous dendritic activity (as measured by EEG) into corticospinal drive (Heitmann et al., 2013), and the innervation of corticospinal projects onto neurons at the spinal level (Harel et al., 2008). In contrast, the synaptic transformations underlying IMC are confined to the spinal motor units (MUs). They involve: (1) the transformation of presynaptic input into the firing patterns of alpha motoneurons and (2) the propagation of the action potential along the muscle fibers resulting in the MU action potential (MUAP). The MUAP itself is a composite potential and is affected by physiological changes due to exercise, fatigue, aging, and disease (McGill et al., 2001). Since changes in the MUAP and the firing rate affect the level of IMC (Boonstra and Breakspear, 2012; Farina et al., 2013), these factors need to 
be controlled for when comparing IMC across conditions.

New techniques may reduce some of these peripheral effects on CMC and IMC, e.g., high-pass filtering EMG before rectification may diminish the effect of EMG signal cancellation (Boonstra and Breakspear, 2012; Farina et al., 2013). In addition, high-density surface EMG (HDsEMG) has been used to improve force estimation (Staudenmann et al., 2010) and may also improve the estimation of common oscillatory input. In particular, HDsEMG signals can be decomposed into spike train patterns using advanced analysis techniques (Holobar et al., 2009). This approach inverts the second transformation concerning the MUAPs and reduces the problem to estimating the common input based on the motoneuron spike trains (Halliday et al., 1995). Assuming that the input is common to all motoneurons, this transmission is largely linear and a few motoneurons will accurately sample the input (Negro and Farina, 2011). However, physiological states like fatigue are also known to affect synaptic transmission (Gandevia, 2001), and hence affect how motoneurons sample the input. This problem may be overcome by model inversion techniques that are used to infer the common input from multiple neural spike-train data (Kulkarni and Paninski, 2007; Paninski et al., 2007). These models can be validated using experimental data where the source of common input is known, e.g., when generated by transcranial magnetic stimulation (Norton and Gorassini, 2006).

In sum, whilst the concerns expressed by Heroux and Gandevia (2013) regarding the ambiguity of the underlying mechanisms are valid, the potential of CMC and IMC to investigate new aspects in human motor control warrants further research. Novel techniques such as HDsEMG and model inversion can be used to obtain a more accurate estimate of common input and facilitate the comparison of CMC and IMC between different recording conditions.

\section{ACKNOWLEDGMENTS}

$\mathrm{TB}$ is the recipient of a Postdoctoral Fellowship from the Netherlands Organization for Scientific Research, Veni scheme (NWO \#45110-030).

\section{REFERENCES}

Bernstein, N. A. (1967). The Co-ordination and Regulation of Movements. Oxford: Pergamon Press.

Boonstra, T. W., and Breakspear, M. (2012). Neural mechanisms of intermuscular coherence: implications for the rectification of surface electromyography. J. Neurophysiol. 107, 796-807. doi: 10.1152/jn.00066.2011

Boonstra, T. W., Daffertshofer, A., Roerdink, M., Flipse, I., Groenewoud, K., and Beek, P. J. (2009a). Bilateral motor unit synchronization of leg muscles during a simple dynamic balance task. Eur. J. Neurosci. 29, 613-622. doi: 10.1111/j.14609568.2008.06584.x

Boonstra, T. W., van Wijk, B. C., Praamstra, P., and Daffertshofer, A. (2009b). Corticomuscular and bilateral EMG coherence reflect distinct aspects of neural synchronization. Neurosci. Lett. 463, 17-21. doi: 10.1016/j.neulet.2009.07.043

Farina, D., Negro, F., and Jiang, N. (2013). Identification of common synaptic inputs to motor neurons from the rectified electromyogram. J. Physiol. 591, 2403-2418. doi: 10.1113/jphysiol.2012.246082

Farmer, S. F. (1998). Rhythmicity, synchronization and binding in human and primate motor systems. J. Physiol. 509 (pt 1), 3-14. doi: 10.1111/j.14697793.1998.003bo.x

Friston, K. J., Harrison, L., and Penny, W. (2003). Dynamic causal modelling. Neuroimage 19, 1273-1302. doi: 10.1016/S1053-8119(03)00202-7

Gandevia, S. C. (2001). Spinal and supraspinal factors in human muscle fatigue. Physiol. Rev. 81, 1725-1789.

Halliday, D. M., Rosenberg, J. R., Amjad, A. M., Breeze, P., Conway, B. A., and Farmer, S. F. (1995). A framework for the analysis of mixed time series/point process data-theory and application to the study of physiological tremor, single motor unit discharges and electromyograms. Prog. Biophys. Mol. Biol. 64, 237-278. doi: 10.1016/S0079-6107(96) 00009-0

Harel, R., Asher, I., Cohen, O., Israel, Z., Shalit, U., Yanai, Y., et al. (2008). Computation in spinal circuitry: lessons from behaving primates. Behav. Brain Res. 194, 119-128. doi: 10.1016/j.bbr.2008.07.013

Heitmann, S., Boonstra, T., and Breakspear, M. (2013). A dendritic mechanism for decoding traveling waves: principles and applications to motor cortex. PLoS Comput. Biol. 9:e1003260. doi: 10.1371/journal.pcbi.1003260

Heroux, M. E., and Gandevia, S. C. (2013). Human muscle fatigue, eccentric damage and coherence in the EMG. Acta Physiol. (Oxf) 208, 294-295. doi: 10.1111/apha.12133

Holobar, A., Farina, D., Gazzoni, M., Merletti, R., and Zazula, D. (2009). Estimating motor unit discharge patterns from high-density surface electromyogram. Clin. Neurophysiol. 120, 551-562. doi: 10.1016/j.clinph.2008.10.160

Kulkarni, J. E., and Paninski, L. (2007). Common-input models for multiple neural spike-train data. Network 18, 375-407. doi: 10.1080/09548980701625173

McGill, K. C., Lateva, Z. C., and Xiao, S. (2001). A model of the muscle action potential for describing the leading edge, terminal wave, and slow afterwave. IEEE Trans. Biomed. Eng. 48, 1357-1365. doi: 10.1109/10.966595

Nazarpour, K., Barnard, A., and Jackson, A. (2012). Flexible cortical control of task-specific muscle synergies. J. Neurosci. 32, 12349-12360. doi: 10.1523/jneurosci.5481-11.2012

Negro, F., and Farina, D. (2011). Linear transmission of cortical oscillations to the neural drive to muscles is mediated by common projections to populations of motoneurons in humans. J. Physiol. 589, 629-637. doi: 10.1113/jphysiol.2010.202473

Norton, J. A., and Gorassini, M. A. (2006). Changes in cortically related intermuscular coherence accompanying improvements in locomotor skills in incomplete spinal cord injury. J. Neurophysiol. 95, 2580-2589. doi: 10.1152/jn.01289.2005

Paninski, L., Pillow, J., and Lewi, J. (2007). Statistical models for neural encoding, decoding, and optimal stimulus design. Prog. Brain Res. 165, 493-507. doi: 10.1016/S0079-6123(06)65031-0

Poston, B., Danna-Dos Santos, A., Jesunathadas, M., Hamm, T. M., and Santello, M. (2010). Force-independent distribution of correlated neural inputs to hand muscles during three-digit grasping. J. Neurophysiol. 104, 1141-1154. doi: 10.1152/jn.00185.2010

Semmler, J. G., Ebert, S. A., and Amarasena, J. (2013). Eccentric muscle damage increases intermuscular coherence during a fatiguing isometric contraction. Acta Physiol. (Oxf) 208, 362-375. doi: 10.1111/apha.12111

Staudenmann, D., Roeleveld, K., Stegeman, D. F., and van Dieen, J. H. (2010). Methodological aspects of SEMG recordings for force estimation-a tutorial and review. J. Electromyogr. Kinesiol. 20, 375-387. doi: 10.1016/j.jelekin.2009.08.005

Todorov, E. (2004). Optimality principles in sensorimotor control. Nat. Neurosci. 7, 907-915. doi: 10.1038/nn1309

Tresch, M. C., and Jarc, A. (2009). The case for and against muscle synergies. Curr. Opin. Neurobiol. 19, 601-607. doi: 10.1016/j.conb.2009.09.002

Ward, N. J., Farmer, S. F., Berthouze, L., and Halliday, D. M. (2013). Rectification of EMG improves detection of motor unit coherence in the beta frequency band. J. Neurophysiol. 110, 1744-1750. doi: 10.1152/jn.00296.2013

Williams, E. R., Soteropoulos, D. S., and Baker, S. N. (2010). Spinal interneuron circuits reduce approximately $10-\mathrm{Hz}$ movement discontinuities by phase cancellation. Proc. Natl. Acad. Sci. U.S.A. 107, 11098-11103. doi: 10.1073/pnas.0913373107

Received: 26 August 2013; accepted: 22 November 2013; published online: 10 December 2013.

Citation: Boonstra TW (2013) The potential of corticomuscular and intermuscular coherence for research on human motor control. Front. Hum. Neurosci. 7:855. doi: 10.3389/fnhum.2013.00855

This article was submitted to the journal Frontiers in Human Neuroscience.

Copyright (c) 2013 Boonstra. This is an open-access article distributed under the terms of the Creative Commons Attribution License (CC BY). The use, distribution or reproduction in other forums is permitted, provided the original author(s) or licensor are credited and that the original publication in this journal is cited, in accordance with accepted academic practice. No use, distribution or reproduction is permitted which does not comply with these terms. 